\title{
PROBABILITY FOR THE FORMATION OF COMPLEX RING MOLECULES IN INTERSTELLAR MEDIUM AND THEIR DETECTION PROPOSAL
}

\author{
K.K.Ghosh \\ Indian Institute of Astrophysics, Kavalur Observatory
}

\begin{abstract}
The pathways for the formation and destruction processes of ring molecules in interstellar medium have been proposed and their abundances have been calculated under steady state conditions and calculated abundances are of the order of $10^{-12} n(H)$, where $n(H)$ is the abundance of hydrogen. Also their detection proposal has been presented.
\end{abstract}

Radioastronomical observations of microwave emission spectra have led to the discovery of many molecules including long-chain hydro-carbons in interstellar medium (ISM). Most of the molecular species appear to be located in dense interstellar clouds that are light years in extent and posses gaseous densities in the range $10^{3}-10^{6}$ molecules $\mathrm{cm}^{-3}$ and have temperature below $100 \mathrm{~K}$. Dust grains associated with the gas scatter and absorb stellar radiation so that molecules in dense clouds are protected from photodissociation. Elemental abundançes in dense clouds are (Allen 1973): $\mathrm{H}(1.0)$, $\mathrm{He}(0.09)$, $O\left(7 \times 10^{-4}\right), C\left(3 \times 10^{-5}\right)$ and $\mathrm{N}\left(9 \times 10^{-5}\right)$. Hydrogen exists in ISM mainly in its molecular form ( $\mathrm{H}_{2}$. Hydrogen atoms are converted to $\mathrm{H}_{2}$ molecules on the surface of dust grains because no competitive gas-phase mechanism has been found. Ionisation of $\mathrm{H}_{2}$ molecules is caused by cosmic rays impinging on dense clouds. These $\mathrm{H}_{2}^{+}$molecules then help for the formation of polyatomic molecules through gas-phase ion-molecular reactions (Mitchell et al. 1978, Prassad \& Huntress, 1980). Interstellar ion-molecule chemistry model can explain the formation and destruction processes of long-chain hydro-carbons. However no detailed studies have been carried out for the formation and destruction processes of ring molecules in ISM (Ghosh \& Ghosh 1980 and 1985). Though no ring molecules have been detected in ISM but the infrared unidentified emission bands $(3.28,3.4,3.5,6.2,7.7,8.6$ and $11.3 \mu \mathrm{m})$ observed in a wide variety of celestial objects (Aitken 1981, Willner 1984, Bregman et al. 1983) have been identified as the polycyclic aromatic hydrocarbons which exists in solid state on the interstellar grain surfaces (Dulley and Williams, 1981; Sakata et al. 1984 and Crawford et al. 1985). On the basis of these results, attempts have been made to develop the pathways for the formation and destruction processes of ring molecules in ISM through gas-phase ion-molecule reactions followed by dissociative electron recombination processes. Also the abundances of these molecules have been calculated 
under steady-state condition. Green and Herbst (1979) have pointed out that many metastable isomers of stable molecules analogous to the case of $\mathrm{HNC}$ and $\mathrm{HCN}$ probably have observable abundances in interstellar dark clouds and many of these compounds may be intermediates in important organic reactions. But they have suggested a few metastable isomers which help to find out the pathways for the formation of open chain molecules. On the basis of theoretical calculations it has been found that the following compound<smiles>N#CC(N)=C(N)C#N</smiles>

is an important metastable isomer which helps to form ring molecules from open chain molecules, in ISM. The main destruction processes of these molecules may be the reactions with $\mathrm{C}^{\boldsymbol{t}}$ ions. The abundances of these molecules have been calculated under steady state condition and the calculated abundances are of the order of $10^{-12} \mathrm{n}(\mathrm{H})$ where $\mathrm{n}(\mathrm{H})$ is the abundance of hydrogen. Regarding the detection of ring molecules in ISM it is important to carryout laboratory experiments to synthesize the ring molecules in gas-phase and high resolution IR spectra of those molecules are to be obtained and then searches are to be made in ISM.

\section{$\underline{\text { References }}$}

Aitken,D.K., 1981 in IAU Symposium 96, Infrared Astronomy, ed. C.G.WynnWillians and D.P. Cruikshank (D.Reidel), 207.

Allen, C.W., 1973: Astrophysical Quantities (3rd ed. : London: Athlone). Bregman, J.D., Dinerstein, H.L., Goebel, J.H., Lester, D.F., Witteborn, F.C. and Rank, D.M., 1983,Ap.J., 274, 666.

Crawford, M.K., Tielens, A.G.G. and Allamandola,L.J., 1985, Ap. J. Letts., 293, L45.

Dulley. W.W. and Williams, D.A., 1981, Mon. Not. Roy. Astrn. Soc., 196,269. Ghosh, K.K. and Ghosh, S.N., 1980, COSPAR Life Sci. Space Res., 18, 37. Ghosh, K.K., 1985, Chem. Phys. Lett. 114, 393.

Mitchell, G., Ginzburg, J.L. and Kuntz, P.J., 1978, Ap.J. Suppl., 38, 39. Prassad, S.S. and Huntress, W.T., 1980, Ap. J. Suppl., 43, 1.

Sakata, A., Wada, S., Tanabe, T. and Onaka, T., 1984, Ap. J. Letts., 287, L 51. Willner,S.P., 1984, in Galactic and Extragalactic Infrared Spectroscopy, ed. M.F. Kessler and J.P. Phillips (D. Reidel), 37. 